\title{
Resection of biliary mucinous cystic neoplasms of the liver: a prospective cohort series of 13 consecutive patients
}

\author{
JC Kloppers, JEJ Krige, MM Bernon, S Burmeister, E Jonas, SR Thomson, PC Bornman \\ Surgical and Medical Gastroenterology Units, Departments of Surgery and Medicine, University of Cape Town Health Scienc- \\ es Faculty, Groote Schuur Hospital
}

Corresponding author: JC Kloppers (christo.kloppers@uct.ac.za)

\begin{abstract}
Background: Biliary mucinous cystic neoplasms (BMCNs) are uncommon neoplastic septated intrahepatic cysts which are often incorrectly diagnosed and have the potential for malignant transformation.

Objectives: To assess the outcome of surgical resection of BMCNs.

Methods: A prospective liver surgery database was used to identify patients who underwent surgery at Groote Schuur Hospital Complex for BMCN from 1999 to 2015. Demographic variables including age and gender were documented as well as detailed preoperative imaging, location and size, operative treatment, extent of resection, histology, postoperative complications and outcome.

Results: Thirteen female patients (median age 45 years) had surgery. Eleven were diagnosed by imaging for symptoms. Two were jaundiced. One cyst was found during an elective cholecystectomy. Five cysts were located centrally in the liver. Before referral three cysts were treated with percutaneous drainage and two were treated with operative deroofing. Six patients had anatomical liver resections and seven patients had non anatomical liver resections of which two needed ablation of residual cyst wall. One patient needed a biliary-enteric reconstruction to treat a fistula. Median operative time was 183 minutes (range: 130-375). No invasive carcinoma was found. There was no operative mortality. One surgical site infection and one intra-abdominal collection were treated. Two patients developed recurrent BMCN after 24 months.

Conclusion: BMCNs should be considered in middle aged women who have well encapsulated multilocular liver cysts. Treatment of large central BMCNs adjacent to vascular and biliary structures may require technically complex liver resections and are best managed in a specialised hepato-pancreatico-biliary unit.

Key words: Biliary mucinous cystic neoplasm, biliary cystadenoma, liver cysts, benign liver tumours
\end{abstract}

S Afr J Surg 2018;56(2)

http://dx.doi.org/10.17159/2078-5151/2018/v56n2a2446

\section{Introduction}

Biliary mucinous cystic neoplasms (BMCNs) are uncommon multilocular hepatic cysts which account for less than $5 \%$ of liver cysts. ${ }^{1}$ BMCNs are often incorrectly diagnosed and have the potential for malignant transformation. ${ }^{2}$ Clear guidelines are scant and publications are limited to small case series of BMCNs. ${ }^{3,4}$ Most occur in middle-aged women. Patients may be asymptomatic and the tumours are often discovered incidentally during imaging or at laparotomy. Management is complicated because accurate identification of the invasive form before surgery is seldom possible. The potential for malignant transformation and the tendency to recur, particularly when treated with techniques other than complete excision, are of concern in these tumours. In this study we describe the surgical treatment of 13 patients with BMCNs from a single tertiary centre.

\section{Materials and Methods}

A retrospective analysis of a prospectively maintained database of all liver surgery at Groote Schuur and the University of Cape Town Private Academic Hospitals between 1999 and 2015 was performed to identify all patients with a histological confirmed BMCN, based on the WHO diagnostic criteria. $^{5}$ Patient demographics, clinical presentation and detailed preoperative imaging characteristics of the tumour, including intrahepatic location were recorded. The extent of the surgical procedure was detailed and included operative time and blood transfusion requirements. Postoperative complications were documented according to the ClavienDindo (CD) classification. ${ }^{6}$ Patients were planned for routine six-monthly follow-up. Initial surveillance investigation was abdominal ultrasound and additional investigations, for example CT or MRI were performed as clinically indicated. Descriptive analyses were used to characterise the study 
population. The registry for liver resections (R024/2014) and the analysis of this subgroup (735/2016) were approved by the Faculty of Health Sciences Human Research Ethics Committee of the University of Cape Town.

\section{Results}

During the study period 457 liver resections were performed of which 13 were for BMCN. All 13 were females with a median age of 45 years (range 22-74). The clinical characteristics are summarised in Table 1. Ten patients presented with abdominal pain, eight had a palpable mass and two were jaundiced. In 12 patients the presumptive diagnosis was made on preoperative imaging ( $\mathrm{CT}=11$, US $=1$ ) while one cyst was found incidentally during an elective laparoscopic cholecystectomy (Figure 1). Median cyst size was $13 \mathrm{~cm}$ (range $2.5-30 \mathrm{~cm}$ ) and most occurred centrally in the liver, abutting the hilar bilio-vascular structures. Three patients had percutaneous aspiration of cysts before referral. In two patients inappropriate deroofing was performed before referral. In one, treated as a simple cyst, histology confirmed a $\mathrm{BMCN}$, while in the other a persistent bile leak after deroofing required a formal liver resection.

The operative technique and procedure were adapted to the cyst size and location and proximity of the cyst to vital structures in the porta hepatis, the hepatic veins and the inferior vena cava (Table 2) (Figure 2). In two patients in whom the back wall of the BMCN was adherent to major vascular structures, as much of the cyst wall as could be safely resected was removed and the residual inner cyst surface was ablated with argon beam coagulation. In one patient with a complex centrally located cyst in whom the left hepatic duct communicated with the cyst cavity, a biliary-enteric reconstruction with a Roux-en-Y loop was performed. Four patients had bile leaks identified during surgery that were oversewn. Two patients had postoperative complications. In one patient a $\mathrm{CD}$ grade II surgical site infection was successfully treated with antibiotics and in the other a grade IIIa intra-abdominal collection required radiological percutaneous drainage one month after surgery. Four patients had frozen section performed on the cyst wall intraoperatively, all confirming mucinous neoplasms. None of the patients had invasive BMCN on histology (Figure 3).

All patents had a follow-up after surgery, but long term follow-up, more than 1 month, was only possible in 7 patients with a median follow-up of 12 months (range 1-24 months). Two patients developed recurrent disease after 24 months. Both recurrences were diagnosed on CT scan after new symptoms were investigated. One recurred after partial resection with ablation of the remnant cyst wall and declined further surgery. The other who recurred after a formal resection was treated with percutaneous aspiration and ethanol injection because of a high operative risk. She remains recurrence-free after eight years.

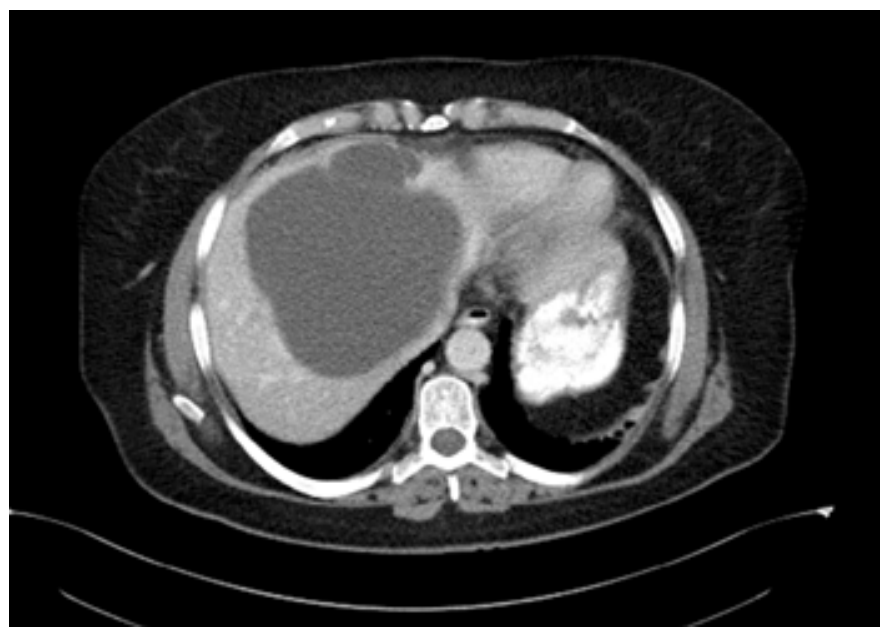

Figure 1. CT axial image of patient 8 demonstrated a typical central placed complex liver cyst

Table 1. Clinical characteristics of patients

\begin{tabular}{ccllcc}
\hline Patient & Age & Symptoms & Clinical Signs & Maximum diameter (cm) & Location (segments) \\
\hline 1 & 46 & RUQ pain & Jaundice \& epigastric mass & 15 & Central $(4,5)$ \\
2 & 47 & N\&V, LOW & Palpable epigastric mass & 25 & Right $(5,6)$ \\
3 & 39 & RUQ pain & None & 2,5 & Right $(5)$ \\
4 & 48 & Epigastric discomfort & Palpable epigastric mass & NA & Central $(2,3,4,5)$ \\
5 & 74 & Epigastric discomfort & None & 12 & Left $(2,3)$ \\
6 & 22 & RUQ pain & Jaundice \& epigastric mass & 10 & Central (4,5) \\
7 & 32 & Biliary colic & None & 21 & Left (3) \\
8 & 45 & Abdominal distention & Palpable epigastric mass & 8 & Central (4,5,6) \\
9 & 35 & RUQ pain & Previous surgery & NA \\
10 & 56 & RUQ pain & Palpable epigastric mass & NA & Right $(5)$ \\
11 & 44 & Epigastric discomfort & NA & 30 & Right $(5,6)$ \\
12 & 38 & Abdominal distention & Palpable epigastric mass & 14 & Central (4,5,8) \\
13 & 63 & Epigastric discomfort & Palpable epigastric mass & & Left $(2,3)$
\end{tabular}




\section{Table 2. Operative procedures $(n=13)$}

\begin{tabular}{ll}
\hline $\begin{array}{l}\text { Operation } \\
\quad \text { Anatomical liver resection }\end{array}$ & 6 \\
$\quad \begin{array}{l}\text { Non-anatomical liver resection } \\
\quad \text { Complete }\end{array}$ & 5 \\
$\quad$ Incomplete resection with ablation & 2 \\
Inflow occlusion & 2 \\
Intra-operative blood transfusion & 1 \\
Intra-operative frozen section performed & 4 \\
Drain & 11 \\
Operative time (minutes), median (range) & $183(130-375)$ \\
ICU stay (days), median (range) & $2(1-3)$ \\
Hospital stay (days), median (range) & $7(5-8)$ \\
\end{tabular}

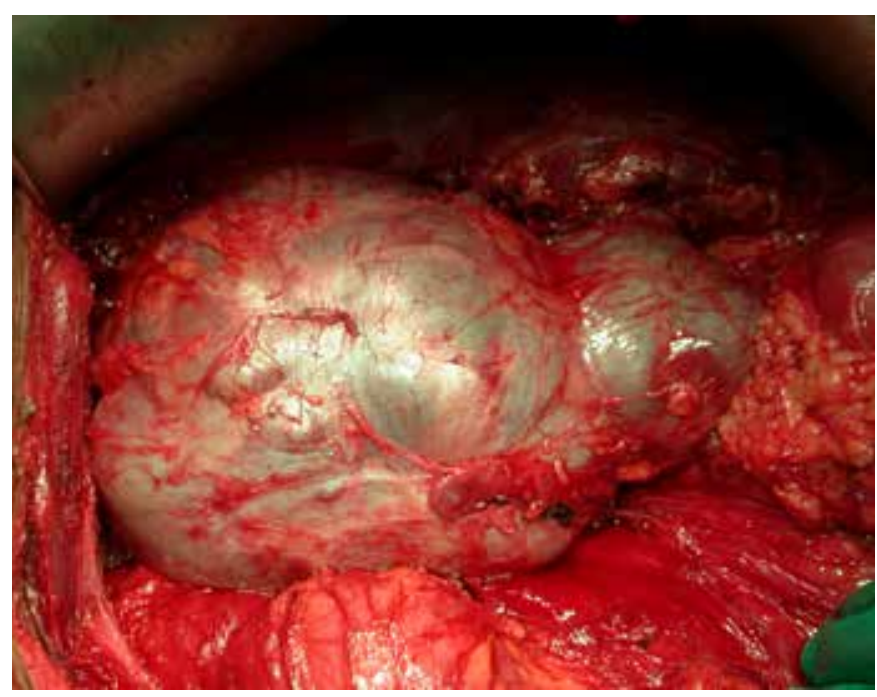

Figure 2. Exposure of BMCN before resection

\section{Discussion}

In this study all patients were females with a median age of 45 years which is in keeping with the literature that reports a female BMCN preponderance of $90 \%$, typically in the fourth and fifth decades of life. ${ }^{1,7}$ The majority of patients presented with cyst-related symptoms as summarised in Table 1. Some BMCNs in our series were mistaken for simple cysts which led to inappropriate initial treatment, including aspiration and deroofing. Patients with incomplete initial resection develop recurrent symptoms and are at risk for malignant transformation, and thus require definitive surgery for complete resection. ${ }^{2,8}$ Older age, male gender and shorter duration of symtoms on presentation are associated with a greater risk of invasive $\mathrm{BMCN} .{ }^{9}$

Abdominal ultrasound typically shows well-demarcated, mostly multilocular hyperechogenic cystic lesions, which may show septae and characteristic papillary projections from the cyst wall. ${ }^{10}$ As in our patients, common features on $\mathrm{CT}$ include low-density, well defined, lobulated, multilocular, thickwalled cystic lesions with internal septa and occasionally mural nodules. ${ }^{5,10} \mathrm{CT}$ also provides additional information regarding the anatomic relation of cysts to major vessels and bile ducts as well as surrounding structures. MRI/MRCP may be of value in assessing proximity to the intrahepatic biliary system and possible biliary communication of cysts, which would make BMCNs unlikely. ${ }^{11}$ By and large, a CT scan will suffice as imaging modality and MRI is unlikely to change management. In most of our patients the typical imaging findings of a large, solitary multiloculated complex liver cyst with internal septation and nodularity was present. Most cysts occurred centrally in the liver, straddling right and left lobes and in close relation to the porta hepatis.

Serum markers were found not to be helpful in the diagnosis in this series, which is in keeping with the results of Wang et al. who in a series of 30 patients found that CA19.9 to be

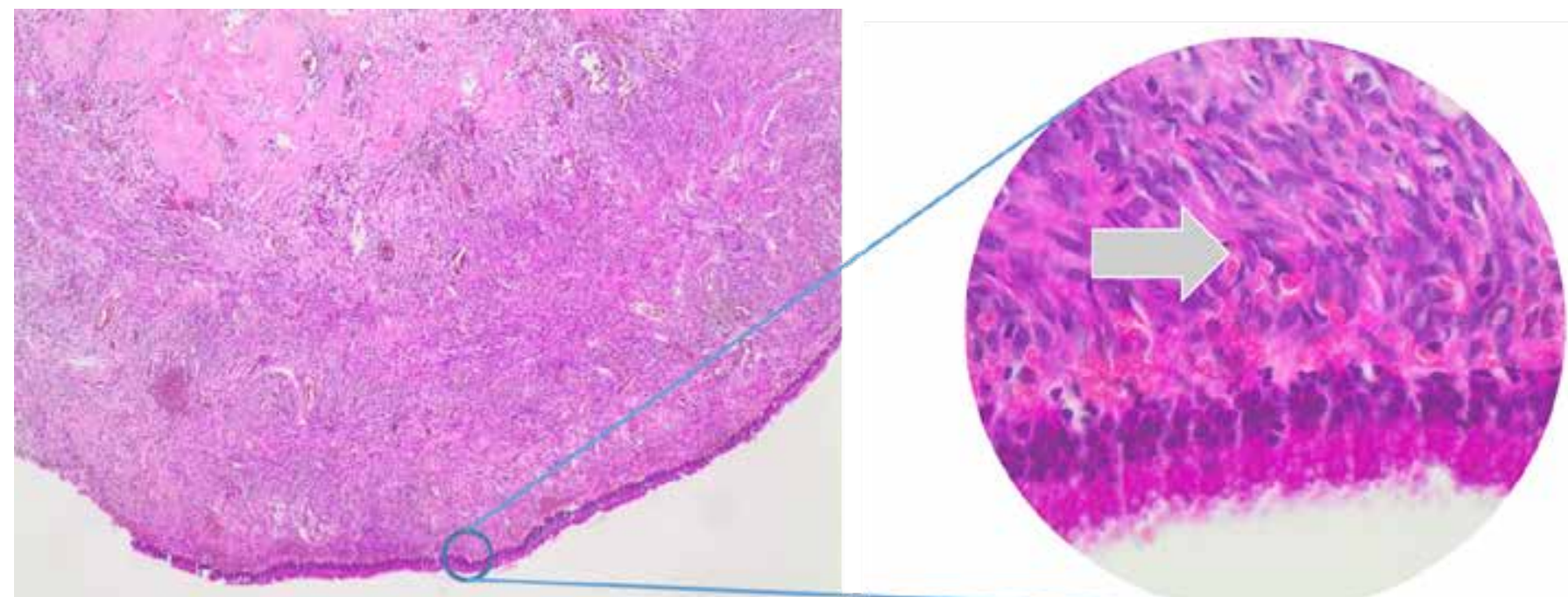

Figure 3. Low and high power histology slides of a BMCN wall with the arrow indicating the ovarian-like stroma 
elevated in only 17 patients. ${ }^{12}$ CA19.9 was available in four of our patients with only one being elevated. Fine needle aspiration with cyst fluid analysis was not used in this current study because of potential harm in disseminating malignant cells and has not proven to change the management plan. ${ }^{11}$

As in our study only a minority of patients present with complications such as jaundice, cyst rupture, bacterial infection or intracystic haemorrhage. Rare complications such as biliary fistulae and inferior vena cava obstruction have been described. ${ }^{4}$ Although all the patients in this study had benign features on histology, malignant change has been reported in up to $10 \%$ of patients. ${ }^{13}$

The only appropriate surgical strategy for treatment of $\mathrm{BMCNs}$ is complete resection with negative margins, either as anatomical or non-anatomical liver resection, depending on the position of the cyst and the relationship to vital structures. The complexity of management is illustrated in a global multi-centre analysis of 248 patients in which $10 \%$ of patients had malignant transformation to invasive BMCNs and $18.3 \%$ recurred after inadequate surgery. ${ }^{13}$ In the largest single-centre cohort of 39 patients, seven were misdiagnosed before initial surgery. Liver resection was performed in 17 patients (eight right and nine left hemi-hepatectomies) and enucleation was performed in 22 patients. At a median followup of 20 months, one patient presented with recurrence. ${ }^{3}$ In our series, six patients had anatomical resections and seven had non-anatomical resections, while two of these needed ablation of residual cyst wall (Table 2). Alternative techniques such as aspiration, internal drainage, intratumoral sclerosant application and incomplete resection result in recurrence rates of $90-100 \%$. Intraoperative frozen section of the cyst wall has not been shown to alter the operative procedure as the biopsy could be non-representative. ${ }^{14}$ There is a single case report of a liver transplant for a central symptomatic $\mathrm{BMCN}$ involving the main vasculobiliary structures. ${ }^{15}$

As in our series, the short-term operative morbidity in published series is low and the prognosis in completely resected BMCNs is excellent. ${ }^{1,12,16}$ Even though recurrence after an adequately treated cyst is less than five percent, follow-up with abdominal ultrasound after resection is recommended.

\section{Conclusion}

BMCNs should be considered in middle-aged women who have well encapsulated multilocular liver cysts. Assessment and treatment of all BMCNs should be in a specialised hepatopancreatico-biliary unit in view of their malignant potential and propensity to recur after resection. Treatment of large central BMCNs adjacent to vascular and biliary structures in particular may require technically complex and challenging liver resections.

\section{Conflicts of interest}

The authors declare no conflict of interest.

\section{REFERENCES:}

1. Simo KA, Mckillop IH, Ahrens WA, Martinie JB, Iannitti DA, Sindram D. Invasive biliary mucinous cystic neoplasm: A review. HPB (Oxford). 2012;14(11):725-40.

2. Martel G, Alsharif J, Aubin JM, et al. The management of hepatobiliary cystadenomas: Lessons learned. HPB (Oxford). 2013;15(8):617-22.

3. Chen YW, Li CH, Liu Z, Dong JH, Zhang WZ, Jiang K. Surgical management of biliary cystadenoma and cystadenocarcinoma of the liver. Genet Mol Res. 2014;13(3):6383-90.

4. Manouras A, Markogiannakis H, Lagoudianakis E, Katergiannakis V. Biliary cystadenoma with mesenchymal stroma: Report of a case and review of the literature. World J Gastroenterol. 2006;12(37):6062-9.

5. Kloppers JC, Krige JEJ, Bernon M, Thomson SR. Nomenclature for neoplastic biliary cysts: Review. South African Gastroenterology Review. 2016;14(1):19-23.

6. Dindo D, Demartines N, Clavien PA. Classification of surgical complications: A new proposal with evaluation in a cohort of 6336 patients and results of a survey. Ann Surg. 2004;240(2):205-13.

7. Kim HH, Hur YH, Koh YS, Cho CK, Kim JW. Intrahepatic biliary cystadenoma: Is there really an almost exclusively female predominance? World J Gastroenterol. 2011;17(25):3073-4.

8. Emre A, Serin KR, Ozden I, et al. Intrahepatic biliary cystic neoplasms: Surgical results of 9 patients and literature review. World J Gastroenterol. 2011;17(3):361-5.

9. Xu MY, Shi XJ, Wan $\mathrm{T}$, et al. Clinicopathological characteristics and prognostic factors of intrahepatic biliary cystadenocarcinoma. Chin Med J (Engl). 2015;128(9):1177-83.

10. Doussot A, Gluskin J, Groot-Koerkamp B, et al. The accuracy of pre-operative imaging in the management of hepatic cysts. HPB (Oxford). 2015;17(10):889-95.

11. Choi HK, Lee JK, Lee KH, et al. Differential diagnosis for intrahepatic biliary cystadenoma and hepatic simple cyst: Significance of cystic fluid analysis and radiologic findings. J Clin Gastroenterol. 2010;44(4):289-93.

12. Wang C, Miao R, Liu H, et al. Intrahepatic biliary cystadenoma and cystadenocarcinoma: An experience of 30 cases. Dig Liver Dis. 2012;44(5):426-31.

13. Arnaoutakis DJ, Kim Y, Pulitano C, et al. Management of biliary cystic tumors: A multi-institutional analysis of a rare liver tumor. Ann Surg. 2015;261(2):361-7.

14. Soares KC, Arnaoutakis DJ, Kamel I, et al. Cystic neoplasms of the liver: Biliary cystadenoma and cystadenocarcinoma. J Am Coll Surg. 2014;218(1):119-28.

15. Ahanatha Pillai S, Velayutham V, Perumal S, et al. Biliary cystadenomas: A case for complete resection. HPB Surg. 2012;2012:501705.

16. Thomas KT, Welch D, Trueblood A, et al. Effective treatment of biliary cystadenoma. Ann Surg. 2005;241(5):769-73; discussion $773-5$. 